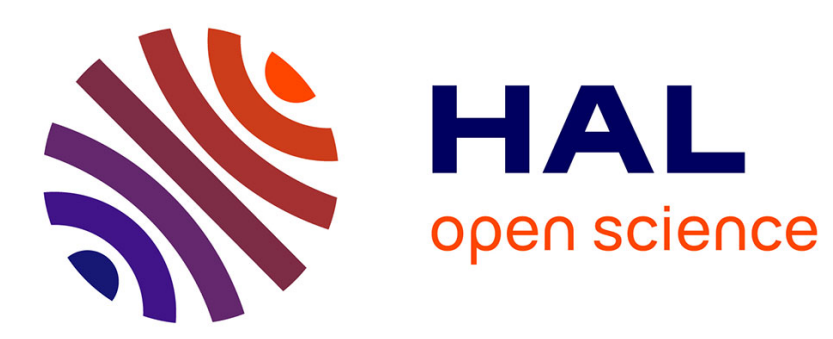

\title{
La souffrance des usagers de drogues incarcérés : regards profanes sur le sens de la peine
}

\author{
Fabrice Fernandez
}

\section{To cite this version:}

Fabrice Fernandez. La souffrance des usagers de drogues incarcérés: regards profanes sur le sens de la peine. ENSP. Normes et valeurs dans le champ de la santé, ENSP, pp. 273-280, 2004, Recherche santé social. hal-00488084

\section{HAL Id: hal-00488084 \\ https://hal.science/hal-00488084}

Submitted on 1 Jun 2010

HAL is a multi-disciplinary open access archive for the deposit and dissemination of scientific research documents, whether they are published or not. The documents may come from teaching and research institutions in France or abroad, or from public or private research centers.
L'archive ouverte pluridisciplinaire HAL, est destinée au dépôt et à la diffusion de documents scientifiques de niveau recherche, publiés ou non, émanant des établissements d'enseignement et de recherche français ou étrangers, des laboratoires publics ou privés. 


\title{
La souffrance des usagers de drogues incarcérés : regards profanes sur le sens de la peine
}

\author{
Fernandez Fabrice, \\ Allocataire de recherche \\ Agence Nationale de Recherche sur le Sida (ANRS), \\ CIRUS (CNRS 5193), Université de Toulouse II
}

Les usages de drogues réguliers et dépendants sont souvent considérés en médecine comme la cause ou la conséquence de souffrances psychiques. Ainsi, le manuel de référence de la psychiatrie Nord américaine (DSM IV), définit la dépendance comme un «mode d'utilisation inadéquat ou inadapté d'une substance conduisant à une altération du fonctionnement ou à une souffrance cliniquement significative ». Mais pour nombre de médecins y compris de généralistes, c'est aussi un «état de souffrance qui conduit à la toxicomanie ». Cette tendance contemporaine à traiter de ce que certains nomment «la nouvelle question sociale» c'est-à-dire de ces individus «désaffiliés », « exclus» et «précaires » surexposés par le manque d'attaches et l'absence de protections (Castel, 1995), par le prisme de la souffrance a été mis à jour dans de nombreux domaines d'intervention sociale (Fassin, 2003, p.18). La prison n'échappe pas à cette tendance mais en concentrant des individus «en souffrance », c'est toute la question du contrôle social et du sens de la peine qu'elle permet au profane de réintérroger. Nous avons recueilli un ensemble de matériaux autour de cette souffrance à la fois à l'intérieur et à l'extérieur de la prison, auprès de sortants de prison, d'usagers de drogues en errance urbaine et de personnes incarcérées en maisons d'arrêt pour infraction à la législation sur les stupéfiants. Il apparaît que l'interprétation du rôle de cette souffrance psychique et de la mise en scène du corps souffrant (automutilation, ingestion d'objet, etc.) oriente les logiques d'actions profanes de manière différenciée et conflictuelle. Mais à travers elles, c'est la question de la place de la souffrance dans ce que punir veut dire (Foucault, 1975) qui est soulevée. 


\section{Accès aux soins et rôle du médicament}

En maisons d'arrêt, les représentations profanes des maladies viennent interférer avec le discours des soignants sur les modes de contamination. Des détenus affirment que «le sida, s'attrape par la sueur » ou la varicelle «par la poussière ». Ces peurs sont accentuées par les difficultés quotidiennes d'accès aux soins (toujours fonction des nécessités et des impératifs du travail des surveillants), ce qui augmente d'autant le climat de méfiance et de suspicion autour du personnel pénitentiaire et médical.

Car la médecine est, elle aussi, considérée comme partie liée avec la peine et les détenus doutent du secret médical et de la confidentialité des dossiers. Pour de nombreux soignants, la pratique médicale participe à la gestion de la détention (Bessin, Lechien, 2001), l'impuissance à établir une véritable relation de soins se traduit dans les discours des détenus comme une volonté tacite de leur nuire. La prison est alors vécue sur le mode de la violence et de la domination, comme une totalité qui cherche à les écarter du jeu social, les paupérise, reproduisant ou amplifiant les inégalités sociales de l'extérieur. Les services médicaux et psychiatriques sont souvent perçus comme faisant partie de ce système de domination et d'exclusion où «les infirmiers qui distribuent des cachets» deviennent «des dealers ambulants » et le médicament, un “outil d'asservissement".

\section{De la plainte à l'imposition d'une norme}

Entre détenus, le discours plaintif reste problématique et devient l'objet d'agacement notamment envers les usagers de drogues dépendants qui sollicitent souvent le personnel médical (notamment pour l'obtention et le suivi de leur traitement de substitution).

Les difficultés quotidiennes pour obtenir un rendez-vous à l'infirmerie accentuent le sentiment «qu'en prison, il n'y en a que pour les drogués». Une approche psychiatrique appréhendant la toxicomanie sur le modèle d'une maladie, est relayée ici par le savoir profane combinant quant à lui, de manière indifférenciée le sida et la toxicomanie. A l'instar de ce détenu qui affirmait «à coté de moi, il y a un mec qui est malade du Sida, on lui donne des cachets de toxicomane », un système d'interprétations et de justifications se met en place associant maladie mentale et maladie physique et permettant d'expliciter les modes d'exclusion envers les usagers de drogues. 
Si l'incompréhension entre détenus naît souvent de situations de décalage vis-à-vis de l'appréhension de la durée de la peine et des modes d'adaptation à mettre en place (les plus problématiques de ces adaptations demeurant le suicide, la tentative de suicide et l'automutilation), en appelant à des incompatibilités d'humeur ou au non-respect des règles fixées par l'administration (ménage, silence, respect, etc.), les logiques d'interaction s'inscrivent dans un véritable contrôle social quotidien visant à la mise à l'écart des « contagieux », (appels répétés aux surveillants, menaces de violence, chantage, etc.).

Les normes qui visent à exclure les usagers de drogue des cellules collectives ou des discussions ordinaires sont présentées comme nécessaires et justes. Le respect qu'on va accorder aux détenus dépendra aussi de leurs capacités à mettre en œuvre ces normes.

\section{Deux modes d'ajustement intérieur/extérieur}

Dés la première incarcération, c'est à un double ajustement que le prévenu est confronté, d'une part, avec le monde extérieur (il doit choisir de se battre ou non pour maintenir son statut, ses rôles, ses liens sociaux, sauver son mariage, son emploi ou ses activités à l'extérieur, obtenir de l'aide, des produits, de l'argent, etc.) mais aussi avec le monde de la prison (choix de ses codétenus, de ses fréquentations, continuer ou stopper sa consommation, travailler, se faire prescrire des produits de substitution ou des médicaments, avoir des activités, etc.). Ainsi l'on peut analyser les comportements des détenus à travers les relations sociales qui s'instaurent en prison. Récemment, C. Rostaing a mis en avant une typologie croisée des logiques d'interaction entre détenues et surveillantes, selon que les premières acceptent ou rejettent leur participation à la logique pénitentiaire et selon que les surveillantes inscrivent leur travail dans une logique missionnaire ou statutaire (Rostaing, 1997). Mais les modes de double ajustement intérieur/extérieur éclairent aussi les différentes logiques d'action profanes qui visent à établir des normes de conduites différenciées vis-à-vis de soi et de sa santé. Les modes d'ajustement (la continuité et la coordination) présentés ici ne recouvrent que partiellement les catégorisations de refus et de participation à la logique pénitentiaire, car nous avons affaire à un conflit d'interprétation des comportements pouvant désigner l'une ou l'autre logique d'interaction. Ainsi, c'est le cadre interprétatif des comportements des détenus par eux-mêmes qui est en jeu. 


\section{La continuité}

Certains individus sont conduits à adapter leurs modes de vies avec leurs incarcérations à répétition et décident même parfois de se rendre en prison quand leur état de santé se dégrade. Il s'agit souvent de sous-prolétaires à la rue, errants ou squatters pour qui la prison peut être l'occasion d'une pause dans la consommation de produits, de trouver un abri pour l'hiver, voire de se refaire une santé. Le fonctionnement totalitaire de la prison rentre en résonance avec le celui des centres d'hébergement d'urgence à l'extérieur (Lanzarini, 2000).

Le jeu de continuité prison/errance ne fait qu'entretenir un mode de vie où chaque incarcération les précarise un peu plus. Connus des surveillants et parfois «particulièrement signalés », ils sont rejetés des cellules collectives de manière répétitive. Cet isolement les rend d'autant plus dépendants de la prison (qui les loge, les nourrit, etc.) et du service médical (pour les soins et les traitements de substitution).

Désignés comme «gratteurs » car constamment en train de quémander quelque chose, leur adaptation relève ici de la survie (obtenir des produits toxiques, des cigarettes, de la nourriture ou quelques vêtements).

Ce type d'articulation peut conduire à des pratiques d'automutilation comme chantage au suicide pour obtenir quelques aménagements à leurs conditions de détention (notamment des produits de substitution). Ils revendiquent ainsi une mesure d'exception liée à une situation personnelle dramatique, une souffrance intime que la prison ne fait qu'accentuer et qui peut les conduire à des actes désespérés. Le passage à l'acte auto-agressif donne une visibilité à cette souffrance et investit le corps comme une ultime ressource.

Ils vivent alors une forme d'expérience totale (Good, 1998 et Castel, 1998) produite ici par la perte de la notion de frontières entre intérieur et extérieur, il y a recentrage sur les produits. Les détenus ne répondent plus au courrier, ne rencontrent plus leurs avocats, restent coucher pendant les heures de promenade, saisissent n'importe quelle occasion pour voler leurs codétenus. L'engagement est devenu total.

Mais de plus en plus isolés et dégradés par des consommations multiples et un manque d'hygiène croissant, ils s'immergent dans des pratiques qui ne tiennent plus compte du cadre dans lequel elles s'inscrivent. Pour tenir le coup, c'est-à-dire continuer d'avoir la force de vivre, ils doivent consommer.

C'est à travers un relâchement progressif du sens accordé aux peines de prisons successives, ainsi qu'au souci de soi et de son corps, que l'usager de toxiques perd toute 
perspective sur l'avenir. Etre contaminé par une maladie ne signifie plus rien si elle n'installe pas une souffrance dissociable du manque lié à la consommation de produits.

\section{La coordination}

Certains détenus adoptent une autre forme d'ajustement qui, pensent-ils, leur permettra de maintenir leurs rôles et statuts sociaux à l'extérieur. Ils sont ainsi persuadés de pouvoir retrouver une « activité normale » au sortir de prison.

Ils semblent assimiler les valeurs, les normes et les codes de l'institution (être sans histoire et «serviable», bonne conduite, etc.), se présentant parfois eux-mêmes comme les garants d'un certain ordre social.

On relève chez eux un souci de soi, une attention particulière au corps (sport, musculation, hygiène). L'usage de drogue à l'extérieur étant souvent resté récréatif, le détenu a d'autant moins de difficultés à avoir une attitude critique vis à vis de ceux qui se laissent aller. Le mode coordonné n'est pas celui de l'épanchement, ici on ne se plaint que de ceux qui se plaignent.

On évite les psychiatres, les psychologues que l'on considère souvent comme les «médecins des fous ». On refuse d'être confondu avec ces détenus qui sont «contents d'être là » et on surveille ses fréquentations. Cet auto-contrôle est doublé d'un contrôle social visant à produire des normes de comportements plus strictes.

L'angoisse concernant les maladies contagieuses se cristallise autour des « toxicomanes » et se double d'un jugement de déviance, caractérisé par un usage repérable et considéré dangereux de médicaments, incarnant ainsi la peur de ne plus pouvoir être maître de soi et de ne plus se conduire rationnellement. L'usager de drogue en échos avec les représentations qui lui sont associées (d'individus violents, voleurs, menteurs, etc.) caractérise un retour à l'animalité par l'usage de toxiques et la fin de la sociabilité.

\section{Des normes pour exclure les « irresponsables »}

S'intéresser au point de vue profane, c'est appréhender les mécanismes qui conduisent l'acteur à exploiter les savoirs et les dispositifs sanitaires, médico-sociaux et judiciaires dont il a connaissance, les constituant en ressources ou en contraintes selon les situations. En détention, certains individus essaient d'imposer des normes à l'égard des usagers de drogues, 
en combinant savoir profane et savoir médical pour justifier l'exclusion, exclusion qui est présentée comme la condition nécessaire à leur propre survie. Le contrôle social profane vise alors à exclure «ceux qui se coupent», «qui se droguent» et qui de plus «ont des maladies », ces «irresponsables » qui ont une logique d'action inacceptable et irrationnelle.

Les usagers de drogues sont considérés comme les «bons drogués» d'une administration pénitentiaire et médicale qui cherche à les rendre dociles par les médicaments conduisant ainsi une entreprise indirecte de mortification (dégradation physique, automutilation, suicide).

Certains affirment que si les psychiatres «veulent t'enterrer, ils t'enterrent, par les médicaments ». La méfiance s'instaure vis-à-vis des objectifs supposés de l'institution médicale, soupçonnée de fabriquer ces individus «dociles », «enterrés vivants », qui «errent dans les couloirs », l'air hagard, n'arrivant plus à communiquer.

Mais au-delà de l'exclusion sanitaire, c'est l'obligation de résister à l'oppression carcérale qui est mis en avant. Nombre de détenus se présentent ainsi comme des résistants «anti-médicament» et qualifient les usagers de drogues dépendants de «déchets de la nature », «d'épaves» ou encore de «zombies». Jugés tels de possibles agresseurs, dangereux et irresponsables, ils sont présentés comme l'objet de l'ultime différenciation, celle qui sépare l'humain du non-humain.

\section{Conclusion : l'enjeu de la reconnaissance de la légitimité d'une souffrance}

Si le sentiment général est pour les détenus d'être devenus des numéros, au-delà de leurs différences, notamment de leurs délits et de leurs histoires de vie, cet effacement apparent de leur individualité engendre confusion et sentiment de malaise. Dés lors, chaque détenu va tenter de se désolidariser de l'ensemble, d'échapper à cette uniformisation par le bas. Ainsi il ne s'agit pas vraiment ici de résistance à l'ordre carcéral, mais bien plutôt de stratégies de protection (ou d'auto-préservation) qui lui permettra de ne pas être confondu avec ces êtres qui symbolisent l'infériorité en combinant l'image du « drogué » et du «SDF ». Un système d'auto-contraintes se constitue visant à garder une apparence de normalité, pour être en situation de continuer d'assumer les rôles et les statuts qui sont les siens à l'extérieur. Il s'agit aussi d'élaborer des normes à travers lesquelles il peut se revendiquer comme un individu responsable et productif, capable de se réinsérer (être honnête, travailleur, etc.). 
L'automutilation est ici considérée comme un acte déviant, car son auteur enfreint une règle profane exprimant une obligation dans les relations entre les individus (rester digne, ne pas répandre son sang, être responsable, propre sur soi, respecter les autres), elle constitue alors une manière de se mettre ou d'être mis à l'écart d'une ou plusieurs activités sociales. Par ailleurs, elle fait peser un doute quant à la participation de l'usager de drogue et qui plus est de l'automutilé, à l'ordre même de la rationalité et à la commune humanité.

On les considère à la fois comme des malades mentaux et des «contagieux » qui ont dépassé le seuil limite de l'altération du rapport à autrui ; des êtres encore vivants mais socialement morts, à qui il ne reste que la plainte ou les cris.

A leur façon, les détenus soulèvent un paradoxe qui anime les discours psychiatriques et judiciaires au sujet des usagers de drogues incarcérés que l'on juge responsables de leurs actes et en même temps atteint d'une pathologie psycho-comportementale.

En prison, la conformité d'une conduite est constamment évaluée en fonction certes de l'acte mais aussi de la personnalité de celui qui le commet et de la situation dans laquelle l'action s'inscrit. En cellule, la proximité avec un usager qui s'auto-médicamente à outrance, s'automutile ou tente de se suicider constitue un cadre dans lequel l'altérité se radicalise.

Ces types d'adaptations secondaires (Goffman, 1968) rentrent ainsi en opposition avec les règles profanes fondamentales qui visent à faire perdurer une forme de contrôle social permettant le « vivre ensemble».

Mais ces règles profanes ne sont partagées que par ceux qui peuvent espérer une conditionnelle ou un jugement plus clément en leur faveur et qui ont tout intérêt, en montrant les gages d'une réinsertion à venir, à reprendre à leur compte le discours institutionnel.

A travers ces maisons d'arrêts organisées en blocs où les détenus sont répartis en fonction de leurs origines culturelles, leurs nationalités, voire leurs classes sociales, c'est l'image d'une société qui différencie et hiérarchise selon les origines sociales et les ressources qui transparaît. Une structure où dominations économiques et culturelles se combinent pour exclure les pauvres et les étrangers.

Enserrés entre un modèle de l'ascension sociale par la délinquance et un désir de résistance à une société oppressante par la consommation, les usagers de drogues en prise avec le contrôle social externe et interne à la prison choisissent bien souvent la fuite en avant dans des conduites autodestructrices.

Le problème se dédouble par les réponses sociales qui sont apportées à la précarité : alternant ou combinant approches répressives et «psychologisation» de la pauvreté. Les 
usagers de drogues précaires et errants sont confrontés à l'ambiguïté de la superposition de ces deux approches. Ils ré-interprètent à leur tour ces réponses, recomposent une logique tenant la maladie pour la «faute » (le délit) ou la maladie comme rachetant la « faute ».

Ils investissent le statut de malade comme ressource identitaire pour tenter d'échapper à la réalité présente (la prison) et passée (leurs actes) [Bessin, Lechien, 2002, pp. 361-373], mais ils suscitent aussi volontairement la maladie et/ou la douleur (automutilations, ingestions d'objets, violences auto-retournées, pratiques à risques, etc.) comme pour se défaire de ce processus d'individualisation qui tend de plus en plus à les juger responsables pénalement de leurs actes tout en essayant de les responsabiliser vis-à-vis de leur santé. Déléguée l'entière responsabilité du niveau de dommages acceptés, qu'ils soient sanitaires (contaminations VIH, VHC) et/ou sociaux (jugements, incarcérations) à des usagers de drogues dépendants, qui plus est vivant dans la précarité, revient à considérer ce niveau de dommages comme un choix de vie (Lovell, 2001) et à définitivement vouloir les cantonner dans l'acceptation et la résignation de leurs inégalités de conditions.

\section{Bibliographie}

American Psychiatric Association. (1994), Manuel diagnostique et statistique des troubles

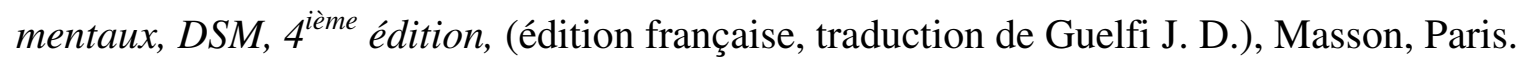

Bessin M., Lechien M-H. (2001), Soignants et malades incarcérés. Conditions, pratiques et usages des soins en prison, CEMS, CES, EHESS.

Castel R. (1998), Les sorties de la toxicomanie, Types, trajectoires, tonalités, Fribourg, Editions universitaires de Fribourg, coll. Res Socialis.

Castel R. (1995), Les métamorphoses de la question sociale, Paris, Fayard, Folio/essais.

Fassin D. (2003), Les nouvelles frontières de la santé, Sciences Humaines, n¹41, aoûtseptembre, pp. 16-24.

Foucault M. (1975), Surveiller et punir : naissance de la prison, Paris, Gallimard.

Good B. (1998), Comment faire de l'anthropologie médicale. Médecine, rationalité et vécu, Le Plessis-Robinson, Les empêcheurs de penser en rond, Institut Synthélabo.

Goffman E. (1968), Asiles. Etudes sur la condition sociale des malades mentaux et autres reclus, Paris, Minuit. 
Lanzarini C. (2000), Survivre dans le monde sous-prolétaire, Paris, PUF.

Lovell A-M. (2001) «Ordonner les risques, l'individu et le pharmaco-sociatif face à l'injection de drogues » in Dozon J-P et Fassin D, Critique de santé publique, Une approche anthropologique, Paris, Balland, Paris, p.340-341.

Rostaing C. (1997), La relation carcérale. Identités et rapports sociaux dans les prisons de femmes, coll. Lien, Paris, PUF. 